Network Working Group

Request for Comments: 1995

Updates: 1035

Category: Standards Track
M. Ohta

Tokyo Institute of Technology

August 1996

Incremental Zone Transfer in DNS

Status of this Memo

This document specifies an Internet standards track protocol for the Internet community, and requests discussion and suggestions for improvements. Please refer to the current edition of the "Internet Official Protocol Standards" (STD 1) for the standardization state and status of this protocol. Distribution of this memo is unlimited.

Abstract

This document proposes extensions to the DNS protocols to provide an incremental zone transfer (IXFR) mechanism.

1. Introduction

For rapid propagation of changes to a DNS database [STD13], it is necessary to reduce latency by actively notifying servers of the change. This is accomplished by the NOTIFY extension of the DNS [NOTIFY].

The current full zone transfer mechanism (AXFR) is not an efficient means to propagate changes to a small part of a zone, as it transfers the entire zone file.

Incremental transfer (IXFR) as proposed is a more efficient mechanism, as it transfers only the changed portion(s) of a zone.

In this document, a secondary name server which requests IXFR is called an IXFR client and a primary or secondary name server which responds to the request is called an IXFR server.

2. Brief Description of the Protocol

If an IXFR client, which likely has an older version of a zone, thinks it needs new information about the zone (typically through SOA refresh timeout or the NOTIFY mechanism), it sends an IXFR message containing the SOA serial number of its, presumably outdated, copy of the zone. 
An IXFR server should keep record of the newest version of the zone and the differences between that copy and several older versions. When an IXFR request with an older version number is received, the IXFR server needs to send only the differences required to make that version current. Alternatively, the server may choose to transfer the entire zone just as in a normal full zone transfer.

When a zone has been updated, it should be saved in stable storage before the new version is used to respond to IXFR (or AXFR) queries. Otherwise, if the server crashes, data which is no longer available may have been distributed to secondary servers, which can cause persistent database inconsistencies.

If an IXFR query with the same or newer version number than that of the server is received, it is replied to with a single SOA record of the server's current version, just as in AXFR.

Transport of a query may be by either UDP or TCP. If an IXFR query is via UDP, the IXFR server may attempt to reply using UDP if the entire response can be contained in a single DNS packet. If the UDP reply does not fit, the query is responded to with a single SOA record of the server's current version to inform the client that a TCP query should be initiated.

Thus, a client should first make an IXFR query using UDP. If the query type is not recognized by the server, an AXFR (preceded by a UDP SOA query) should be tried, ensuring backward compatibility. If the query response is a single packet with the entire new zone, or if the server does not have a newer version than the client, everything is done. Otherwise, a TCP IXFR query should be tried.

To ensure integrity, servers should use UDP checksums for all UDP responses. A cautious client which receives a UDP packet with a checksum value of zero should ignore the result and try a TCP IXFR instead.

The query type value of IXFR assigned by IANA is 251.

3. Query Format

The IXFR query packet format is the same as that of a normal DNS query, but with the query type being IXFR and the authority section containing the SOA record of client's version of the zone. 


\section{Response Format}

If incremental zone transfer is not available, the entire zone is returned. The first and the last $R R$ of the response is the SOA record of the zone. I.e. the behavior is the same as an AXFR response except the query type is IXFR.

If incremental zone transfer is available, one or more difference sequences is returned. The list of difference sequences is preceded and followed by a copy of the server's current version of the SOA.

Each difference sequence represents one update to the zone (one SOA serial change) consisting of deleted RRs and added RRs. The first RR of the deleted RRs is the older SOA RR and the first $R R$ of the added RRs is the newer SOA RR.

Modification of an $R R$ is performed first by removing the original RR and then adding the modified one.

The sequences of differential information are ordered oldest first newest last. Thus, the differential sequences are the history of changes made since the version known by the IXFR client up to the server's current version.

RRs in the incremental transfer messages may be partial. That is, if a single RR of multiple RRs of the same RR type changes, only the changed $R R$ is transferred.

An IXFR client, should only replace an older version with a newer version after all the differences have been successfully processed.

An incremental response is different from that of a non-incremental response in that it begins with two SOA RRs, the server's current SOA followed by the SOA of the client's version which is about to be replaced.

\section{Purging Strategy}

An IXFR server can not be required to hold all previous versions forever and may delete them anytime. In general, there is a trade-off between the size of storage space and the possibility of using IXFR.

Information about older versions should be purged if the total length of an IXFR response would be longer than that of an AXFR response. Given that the purpose of IXFR is to reduce AXFR overhead, this strategy is quite reasonable. The strategy assures that the amount of storage required is at most twice that of the current zone information. 
Information older than the SOA expire period may also be purged.

6. Optional Condensation of Multiple Versions

An IXFR server may optionally condense multiple difference sequences into a single difference sequence, thus, dropping information on intermediate versions.

This may be beneficial if a lot of versions, not all of which are useful, are generated. For example, if multiple ftp servers share a single DNS name and the IP address associated with the name is changed once a minute to balance load between the ftp servers, it is not so important to keep track of all the history of changes.

But, this feature may not be so useful if an IXFR client has access to two IXFR servers: $A$ and $B$, with inconsistent condensation results. The current version of the IXFR client, received from server $A$, may be unknown to server B. In such a case, server B can not provide incremental data from the unknown version and a full zone transfer is necessary

Condensation is completely optional. Clients can't detect from the response whether the server has condensed the reply or not.

For interoperability, IXFR servers, including those without the condensation feature, should not flag an error even if it receives a client's IXFR request with a unknown version number and should, instead, attempt to perform a full zone transfer.

\section{Example}

Given the following three generations of data with the current serial number of 3 ,

JAIN.AD.JP. IN SOA NS.JAIN.AD.JP. mohta.jain.ad.jp. ( $1600600 \quad 3600000604800)$

NS.JAIN.AD.JP. IN A 133.69 .136 .1

NEZU.JAIN.AD.JP. IN A 133.69 .136 .5

NEZU.JAIN.AD.JP. is removed and JAIN-BB.JAIN.AD.JP. is added.

$\begin{array}{lll}\text { jain.ad.jp. } & \text { IN SOA ns.jain.ad.jp. mohta.jain.ad.jp. ( } \\ & \text { IN NS } & \text { NS.JAIN.AD.JP. } \\ \text { NS.JAIN.AD.JP. } & \text { IN A } & 133.69 .136 .1 \\ \text { JAIN-BB.JAIN.AD.JP. } & \text { IN A } & 133.69 .136 .4 \\ & \text { IN A } & 192.41 .197 .2\end{array}$


One of the IP addresses of JAIN-BB.JAIN.AD.JP. is changed.

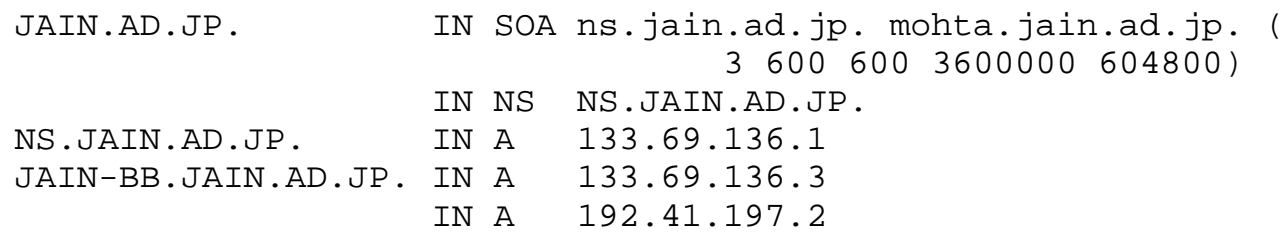

The following IXFR query

\begin{tabular}{|c|c|}
\hline Header & OPCODE =SQUERY \\
\hline Question & QNAME $=J A I N . A D . J P ., Q C L A S S=I N, Q T Y P E=I X F R$ \\
\hline Answer & $<$ empty> \\
\hline Authority & IN $S O A$ serial=1 \\
\hline Additional & $<$ empty $>$ \\
\hline
\end{tabular}

could be replied to with the following full zone transfer message:

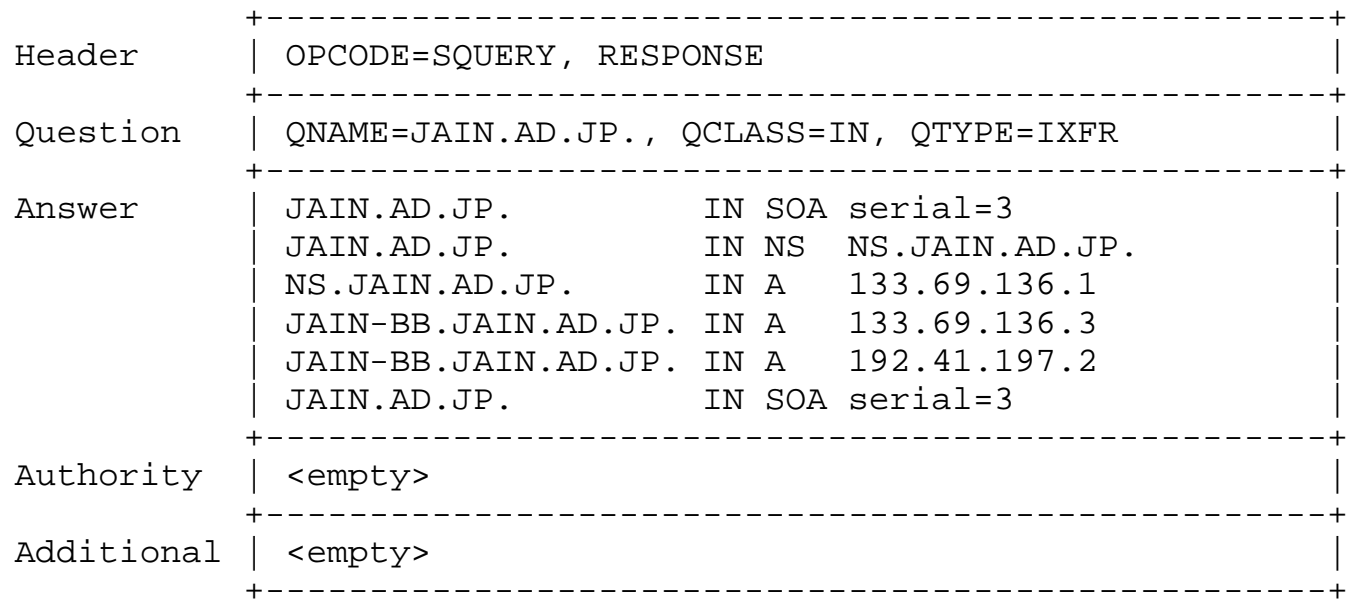


or with the following incremental message:

\begin{tabular}{|c|c|c|}
\hline Header & OP CODE =SQUERY, RESP & ONSE \\
\hline Question & QNAME = JAIN . AD . JP . , & QCLASS=IN, QTYPE=IXFR \\
\hline Answer & 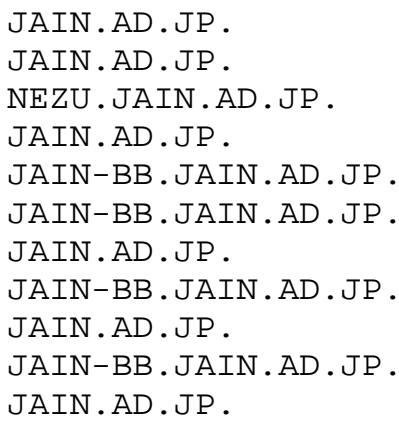 & $\begin{array}{ll}\text { IN } S O A & \text { serial=3 } \\
\text { IN } \text { SOA } & \text { serial=1 } \\
\text { IN A } & 133.69 .136 .5 \\
\text { IN SOA } & \text { serial=2 } \\
\text { IN A } & 133.69 .136 .4 \\
\text { IN A } & 192.41 .197 .2 \\
\text { IN SOA } & \text { serial=2 } \\
\text { IN A } & 133.69 .136 .4 \\
\text { IN SOA } & \text { serial=3 } \\
\text { IN A } & 133.69 .136 .3 \\
\text { IN SOA } & \text { serial=3 }\end{array}$ \\
\hline Authority & <empty > & \\
\hline Additional & $<$ empty> & \\
\hline
\end{tabular}

with the following condensed incremental message:

\begin{tabular}{|c|c|c|}
\hline Header & OPCODE=SQUERY, RESP & ONSE \\
\hline Question & QNAME = JAIN . AD . JP . , & QCLASS=IN, QTYPE=IXFR \\
\hline Answer & $\begin{array}{l}\text { JAIN. AD . JP . } \\
\text { JAIN. AD. JP . } \\
\text { NEZU. JAIN . AD. JP. } \\
\text { JAIN. AD. JP . } \\
\text { JAIN-BB. JAIN. AD. JP. } \\
\text { JAIN-BB. JAIN. AD . JP. } \\
\text { JAIN. AD. JP. }\end{array}$ & $\begin{array}{ll}\text { IN } \text { SOA } & \text { serial=3 } \\
\text { IN } \text { SOA } & \text { serial=1 } \\
\text { IN A } & 133.69 .136 .5 \\
\text { IN SOA } & \text { serial=3 } \\
\text { IN A } & 133.69 .136 .3 \\
\text { IN A } & 192.41 .197 .2 \\
\text { IN SOA } & \text { serial=3 }\end{array}$ \\
\hline Authority & <empty> & \\
\hline Additional & <empty> & \\
\hline
\end{tabular}


or, if UDP packet overflow occurs, with the following message:

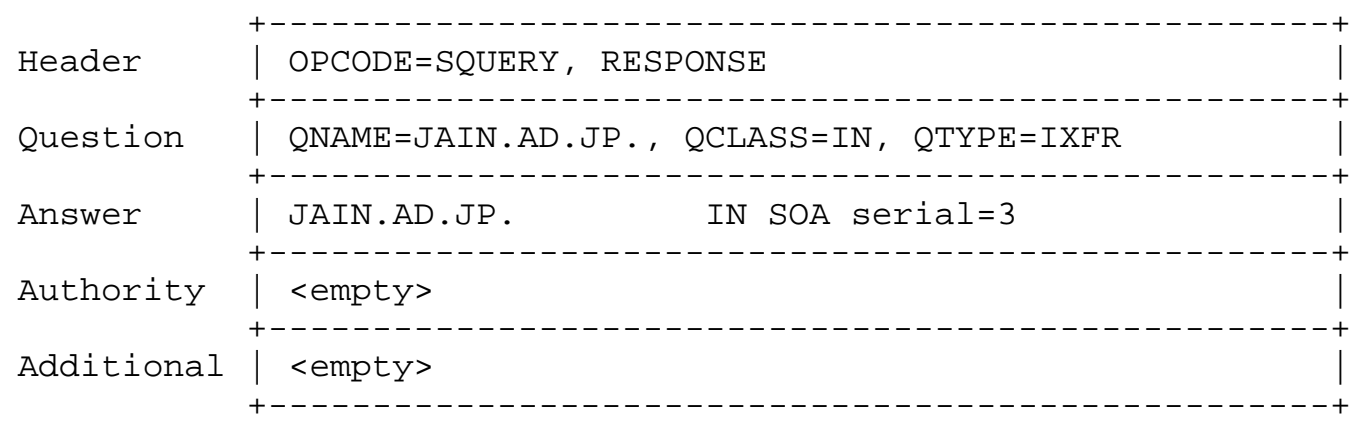

8. Acknowledgements

The original idea of IXFR was conceived by Anant Kumar, Steve Hotz and Jon Postel.

For the refinement of the protocol and documentation, many people have contributed including, but not limited to, Anant Kumar, Robert Austein, Paul Vixie, Randy Bush, Mark Andrews, Robert Elz and the members of the IETF DNSIND working group.

9. References

[NOTIFY] Vixie, P., "DNS NOTIFY: A Mechanism for Prompt Notification of Zone Changes", RFC 1996, August 1996.

[STD13] Mockapetris, P., "Domain Name System", STD 13, RFC 1034 and RFC 1035), November 1987.

10. Security Considerations

Though DNS is related to several security problems, no attempt is made to fix them in this document.

This document is believed to introduce no additional security problems to the current DNS protocol. 
11. Author's Address

Masataka Ohta Computer Center

Tokyo Institute of Technology

2-12-1, O-okayama, Meguro-ku, Tokyo 152, JAPAN

Phone: +81-3-5734-3299

Fax: +81-3-5734-3415

EMail: mohta@necom830.hpcl.titech.ac.jp 